\title{
Conocimiento y práctica de madres sobre alimentación y su relación con el estado nutricional del Programa Nacional Cuna Más en Ciudad Nueva (Tacna, 2019)
}

\section{Knowledge and practice of mothers on food and its relationship with the nutritional status of the Cuna Mas National Program in Ciudad Nueva (Tacna, 2019)}

\author{
Irma Mamani Mayta ${ }^{1 a}$, Elena Cachicatari Vargas ${ }^{1 b}$ \\ ${ }^{1}$ Escuela de Enfermería, Universidad Nacional Jorge Basadre Grohmann. Tacna, Perú. \\ ${ }^{a}$ Bachiller en Enfermería. \\ ${ }^{\text {b }}$ Licenciada en Enfermería, Doctora en Ciencias: Enfermería, Docente Principal.
}

\section{Resumen}

Objetivo: Establecer la relación entre el conocimiento y la práctica de madres sobre alimentación y su relación con el estado nutricional del Programa Nacional Cuna Más en el distrito Ciudad Nueva de Tacna. Material y métodos: Estudio cuantitativo y correlacional. La población estuvo conformada por 50 madres y niños menores de tres años, previo consentimiento informado. Para el análisis estadístico se utilizó estadística descriptiva y la prueba de ji al cuadrado. Resultado: E1 $54 \%$ de madres presentó un nivel de conocimiento alto; el 32\%, medio; y el 14\%, conocimiento bajo. En las prácticas, el $52 \%$ demostró prácticas inadecuadas y el $48.0 \%$ adecuadas. El estado nutricional de los niños; según el indicador peso/talla, el $58.0 \%$ presentó peso normal; el $20 \%$ tiene sobrepeso; el $16 \%$, obesidad; y el $6 \%$, desnutrición. En el indicador peso/edad; el $74 \%$ de niños presentó peso normal; el $20 \%$, sobrepeso; y el $6 \%$, desnutrición. En el indicador talla/edad; el $60 \%$ de niños presentó talla normal para su edad; el $32.0 \%$, talla baja; y solo el $8 \%$, talla alta. Conclusión: Más de la mitad de madres presentó alto nivel de conocimiento; la mayoría presentó prácticas inadecuadas de alimentación; la mayoría de los niños presentó un estado nutricional normal, según indicador peso/talla, peso/edad y talla/edad. Existe relación significativa entre conocimiento y práctica sobre el estado nutricional.

Palabras clave: Conocimiento, práctica, alimentación, madres, estado nutricional.

\begin{abstract}
Aim: To establish the relationship between knowledge and practice of mothers on food and its relationship with the nutritional status of the Cuna Mas National Program in Ciudad Nueva, Tacna. Material and methods: Quantitative and correlational study. The population consisted of 50 mothers and children under three, with prior informed consent. Descriptive statistics and the Chi-square test were used for statistical analysis. Result: $54 \%$ of mothers presented a high level of knowledge, $32 \%$ medium and $14 \%$ low knowledge. In practices $52 \%$ had inadequate practices and $48.0 \%$ adequate. The nutritional status of children according to the Weight / Height indicator $58.0 \%$ had normal weight, $20 \%$ overweight, $16 \%$ obesity and $6 \%$ malnutrition, in the Weight / Age indicator $74 \%$ of children presented normal weight, $20 \%$ overweight and $6 \%$ malnutrition, in the Height / Age indicator $60 \%$ of children presented normal size for their age, $32.0 \%$ short and only $8 \%$ tall. Conclusion: More than half of the mothers presented a high level of knowledge, the majority presented inadequate feeding practices, the majority of the children presented a normal nutritional status according to the Weight / Height, Weight / Age and Height / Age indicator. There is a significant relationship between knowledge and practice on nutritional status.
\end{abstract}

Keywords: Knowledge, practice, feeding, mothers, nutritional status. 


\section{Introducción}

La presente investigación tuvo como objetivo establecer la relación que existe entre el conocimiento y las prácticas alimentarias de las madres y el estado nutricional en niños. En los tres primeros años de vida, una buena alimentación es de vital importancia para el niño, ya que esta etapa se caracteriza por un crecimiento y desarrollo físico y mental (1). Por lo que se hace necesario asegurarle una alimentación suficiente y adecuada para satisfacer sus necesidades nutricionales, prevenir deficiencias, y enfermedades que afecten su crecimiento y desarrollo, así como crear hábitos alimentarios saludables.

Por otro lado; los conocimientos adquiridos y organizados, a través de la experiencia de la madre sobre la alimentación del niño, como la consistencia de las comidas, cantidad, frecuencia y consumo de alimentos de origen animal, influirían en sus prácticas; por lo tanto, esta información es la que nos ayudaría a darle un enfoque real y útil a las intervenciones educativo nutricionales que se realizan en busca de una mejora del estado de salud y nutrición de los niños.

En el Perú, según el patrón de la Organización Mundial de la salud (OMS), la prevalencia de desnutrición crónica infantil (DCI) en menores de 5 años ha disminuido de $28.0 \%$, en el 2017 , a $13.1 \%$, en el 2016 . Siendo las zonas rurales donde se encuentra la mayor proporción de menores de 5 años con desnutrición crónica infantil (2).

Según la Encuesta Demográfica y de Salud Familiar ENDES (2016), en el Perú, la desnutrición crónica es de $13.5 \%$ en niños menores de 5 años, iniciándose entre los 6 a 24 meses de edad, y prácticamente se completa entre los 24 y 36 meses de edad (3).

En Tacna, según la Encuesta Demográfica y de Salud Familiar - ENDES (2016), la desnutrición crónica en menores de 5 años alcanzó el 2.3\%; sin embargo, según estadística de nivel regional, el sistema de información del estado nutricional de niños y gestantes presentan desnutrición crónica, observando que la prevalencia se mantiene con diferencias significativas al interior de los distritos de zonas rurales; la desnutrición global 1\%, desnutrición aguda $1 \%$, sobre peso $12 \%$ y obesidad $3 \%$ (3).

El profesional de enfermería cumple un rol importante en el crecimiento y desarrollo óptimo del niño menor de 3 años, por medio de actividades preventivas y promocionales dirigidas a la madre; con la finalidad de que el niño tenga un crecimiento y desarrollo óptimo de acuerdo a su edad.

\section{Material y métodos}

El presente trabajo de investigación es un enfoque cuantitativo, porque parte de un problema, que una vez delimitado, se derivan objetivos y preguntas de investigación; de las preguntas se establecen hipótesis y se determinan variables; se traza un plan para probarlas (diseño); se miden en un determinado contexto utilizando métodos estadísticos, y se extrae una serie de conclusiones respecto de la hipótesis.

El tipo de investigación de acuerdo a los autores, según Kerlinger, es una investigación descriptiva.

Es correlacional, porque tiene como finalidad conocer la relación, o grado de asociación, que existe entre dos o más variables en una muestra. Es de corte transversal, ya que el método de investigación es de tipo deductivo.

La población de estudio estuvo conformada por 50 madres y niños menores de tres años que asisten al Programa Nacional Cuna Más en el comité 28 del distrito de Ciudad Nueva en Tacna, 2019.

Se incluyó a madres que se encontraron inscritas en el Programa Nacional Cuna Más del distrito de Ciudad Nueva; que tengan hijos menores de tres años, que acepten voluntariamente responder la encuesta, y que dispongan de tiempo necesario para responder el cuestionario. Se excluyeron madres que no estén presentes en el momento de la entrevista.

La validez se realizó mediante 5 expertos especializados en el tema de estudio; se consideró once indicadores de evaluación a juicio de expertos; la validación por criterio tuvo un valor de 93.31, lo cual significa una adecuación total de instrumentos.

A la confiabilidad se realizó una prueba piloto con 10 madres de familia con previo consentimiento informado firmado, y un Alpha de Cronbach con un nivel de conocimiento de 0.664 (confiabilidad marcada).

En el aspecto ético se consideró los principios de autonomía, el consentimiento informado, la confidencialidad de los datos, principio de beneficencia y protección de grupos vulnerables.

\section{Resultados}

En la Tabla 1 se observa que el $42.0 \%$ tienen 1 año y meses de vida; el $38.0 \%$ de 2 años y meses, y tan solo el $20.0 \%$, menos de 1 año de edad. Asimismo, en relación al sexo, tenemos que el $52.0 \%$ es masculino y el $48.0 \%$ femenino.

En la Tabla 2 se observa los datos sociodemográficos de la madre; el $56.0 \%$ tienen entre 21 a 30 años de edad; el $32.0 \%$, entre 31 a 40 años; y el $12.0 \%$, de 41 años a más. En cuanto al número de hijos; el $40.0 \%$ posee un hijo; el $34.0 \%$, dos hijos; el $24.0 \%$, tres hijos, y solo el $2.0 \%$, cuatro hijos. En cuanto al estado civil; el $40.0 \%$ son convivientes; el $28.0 \%$, solteras; el $24.0 \%$; casadas, y el $8.0 \%$, divorciadas. En la ocupación el $62.0 \%$ realiza otras ocupaciones; el $22.0 \%$ trabaja en casa, y solo el $16.0 \%$ es profesional. Finalmente; en cuanto al grado de instrucción encontramos que el $56.0 \%$ posee secundaria completa; el $32.0 \%$, estudios superiores; y $12.0 \%$, estudios primarios.

En la Tabla 3; el 54.0\% tiene un nivel de conocimiento alto sobre la alimentación para menores de tres años; el 32.0\%, un conocimiento mediano; y solo el $14.0 \%$, un 
conocimiento bajo. Asimismo; las prácticas de las madres sobre la alimentación en el menor de tres años, el 52.0\% posee prácticas inadecuadas y el $48.0 \%$ tiene prácticas adecuadas.

En la Tabla 4, el estado nutricional del niño menor de tres años, según el indicador peso/talla; el $58.0 \%$ de infantes presenta peso normal; el $20.0 \%$, sobrepeso; el $16.0 \%$, obesidad; y el $6.0 \%$, desnutrición. En el indicador peso/edad; el $74 \%$ de niños presentan peso normal; 20\%, sobrepeso, y el $6.0 \%$, desnutrición. Por otro lado, en el indicador talla/edad; el $60.0 \%$ de niños presenta talla normal para su edad; el 32,0\% talla baja para su edad, y solo el $8.0 \%$ posee talla alta.

En la Tabla 5, según el indicador peso/talla; el 42.0\% de los infantes con estado nutricional normal poseen madres con un nivel de conocimiento alto; el $16.0 \%$ tienen un estado nutricional normal con madres de nivel de conocimiento medio, y solo el $8.0 \%$ un estado nutricional en sobrepeso con madres de nivel de conocimiento bajo. En el indicador peso/edad observamos; el $52.0 \%$ de los infantes con estado nutricional normal poseen madres con un nivel de conocimiento alto; el $22.0 \%$ poseen un estado nutricional normal con madres de nivel de conocimiento medio, mientras que el $12.0 \%$ un estado nutricional en sobrepeso con madres de nivel de conocimiento bajo. Finalmente, en el indicador talla/edad; el $48.0 \%$ de infantes con una talla normal presentan madres con un nivel de conocimiento alto; el $18.0 \%$ refleja un estado nutricional normal con madres de nivel de conocimiento medio; y el $10.0 \%$, una talla baja con madres de nivel de conocimiento bajo.

En la Tabla 6, según el indicador peso/talla; el 36.0\% de los niños con estado nutricional normal tienen madres que realizan prácticas adecuadas, y el $16.0 \%$ con obesidad tienen madres que realizan prácticas inadecuadas. En el indicador peso/edad; el $44.0 \%$ de los niños con estado nutricional normal tienen madres que realizan prácticas adecuadas, y $18.0 \%$ con sobrepeso tienen madres que realizan prácticas inadecuadas. Finalmente, en el indicador talla/edad; el $36.0 \%$ de niños con una talla normal tienen madres que realizan prácticas adecuadas de alimentación y solo $20.0 \%$ con talla baja tienen madres que realizan prácticas inadecuadas de alimentación.

Tabla 1. Datos sociodemográficos de los niños menores de tres años del Programa Cuna Más de Ciudad Nueva (Tacna-2019).

\begin{tabular}{cccc}
\hline \multicolumn{1}{c}{$\begin{array}{c}\text { Datos } \\
\text { sociodemográficos }\end{array}$} & $\mathbf{N}^{\circ}$ & $\%$ \\
\hline $\begin{array}{c}\text { Menor de 1 } \\
\text { año }\end{array}$ & 10 & 20.0 \\
Edad & $\begin{array}{c}\text { De1 año y } \\
\text { meses } \\
\text { De 2 años y } \\
\text { meses }\end{array}$ & 21 & 42.0 \\
\hline Sexo & 19 & 38.0 \\
\hline $\begin{array}{c}\text { Masculino } \\
\text { Femenino } \\
\text { Total }\end{array}$ & 26 & 52.0 \\
\hline
\end{tabular}

Tabla 2. Datos sociodemográficos de las madres del Programa Cuna Más de Ciudad Nueva (Tacna - 2019).

\begin{tabular}{|c|c|c|c|}
\hline \multicolumn{2}{|c|}{$\begin{array}{l}\text { Datos } \\
\text { sociodemográficos }\end{array}$} & $\mathbf{N}^{0}$ & $\%$ \\
\hline \multirow{3}{*}{ Edad } & $\begin{array}{l}\text { De } 21 \text { a } 30 \\
\text { años }\end{array}$ & 28 & 56.0 \\
\hline & $\begin{array}{l}\text { De } 31 \text { a } 40 \\
\text { años }\end{array}$ & 16 & 32.0 \\
\hline & $\begin{array}{l}\text { De } 41 \text { a más } \\
\text { años }\end{array}$ & 6 & 12.0 \\
\hline \multirow{4}{*}{$\begin{array}{l}\text { Númer } \\
\text { o de } \\
\text { hijos }\end{array}$} & Un Hijo & 20 & 40.0 \\
\hline & Dos Hijos & 17 & 34.0 \\
\hline & Tres hijos & 12 & 24.0 \\
\hline & $\begin{array}{l}\text { Cuatro } \\
\text { Hijos }\end{array}$ & 1 & 2.0 \\
\hline \multirow{4}{*}{$\begin{array}{c}\text { Estado } \\
\text { civil }\end{array}$} & Soltera & 14 & 28.0 \\
\hline & Casada & 12 & 24.0 \\
\hline & Conviviente & 20 & 40.0 \\
\hline & Divorciada & 4 & 8.0 \\
\hline \multirow{3}{*}{$\begin{array}{c}\text { Ocupac } \\
\text { ión }\end{array}$} & Su casa & 11 & 22.0 \\
\hline & Profesional & 8 & 16.0 \\
\hline & Otro & 31 & 62.0 \\
\hline \multirow{4}{*}{$\begin{array}{l}\text { Grado } \\
\text { de } \\
\text { instrucc } \\
\text { ión }\end{array}$} & Primaria & 6 & 12.0 \\
\hline & Secundaria & 28 & 56.0 \\
\hline & Superior & 16 & 32.0 \\
\hline & Total & 50 & 100.0 \\
\hline
\end{tabular}

Tabla 3. Nivel de conocimiento y prácticas de las madres sobre alimentación del Programa Cuna Más de Ciudad Nueva (Tacna - 2019).

\begin{tabular}{cccc}
\hline \multirow{3}{*}{ Conocimiento y prácticas } & $\mathbf{N}^{0}$ & $\%$ \\
\hline \multirow{3}{*}{ Prácticas } & Bajo & 7 & 14.0 \\
& Medio & 16 & 32.0 \\
& Alto & 27 & 54.0 \\
\hline & Inadecuadas & 26 & 52.0 \\
& Adecuadas & 24 & 48.0 \\
& Total & 50 & 100.0 \\
\hline
\end{tabular}

Tabla 4. Estado nutricional en niños menores de tres años del Programa Cuna Más de Ciudad Nueva (Tacna 2019).

\begin{tabular}{cccc} 
Estado & nutricional & $\mathbf{N}^{\circ}$ & $\%$ \\
Peso & Desnutrición & 3 & 6.0 \\
$/$ & Normal & 29 & 58.0 \\
Talla & Sobrepeso & 10 & 20.0 \\
& Obesidad & 8 & 16.0 \\
& TOTAL & 50 & 100.0 \\
\hline \multirow{2}{*}{ Peso } & Desnutrición & 3 & 6.0 \\
$/$ & Normal & 37 & 74.0 \\
Edad & Sobrepeso & 10 & 20.0 \\
& TOTAL & 50 & 100.0 \\
\hline \multirow{2}{*}{ Talla } & Talla Baja & 16 & 32.0 \\
$/$ & Normal & 30 & 60.0 \\
Edad & Talla Alta & 4 & 8.0 \\
& TOTAL & 50 & 100.0 \\
\hline
\end{tabular}


Tabla 5. Conocimiento de madres sobre alimentación y el estado nutricional del Programa Cuna Más de Ciudad Nueva (Tacna-2019).

\begin{tabular}{|c|c|c|c|c|c|c|c|c|c|c|c|}
\hline \multirow{3}{*}{ CONOCIMIENTO } & \multicolumn{8}{|c|}{ Peso / Talla } & \multirow{2}{*}{\multicolumn{2}{|c|}{ TOTAL }} & \multirow{3}{*}{$z$} \\
\hline & \multicolumn{2}{|c|}{ Desnutrición } & \multicolumn{2}{|c|}{ Normal } & \multicolumn{2}{|c|}{ Sobrepeso } & \multicolumn{2}{|c|}{ Obesidad } & & & \\
\hline & $\mathrm{N}^{\circ}$ & $\%$ & $\mathbf{N}^{0}$ & $\%$ & $\mathrm{~N}^{*}$ & $\%$ & $\mathbf{N}^{\circ}$ & $\%$ & $\mathrm{~N}^{0}$ & $\%$ & \\
\hline Baio & 1 & 2,0 & 0 & 0.0 & 4 & 8,0 & 2 & 4,0 & 7 & 14,0 & \\
\hline Medio & 2 & 4,0 & 8 & 16,0 & 3 & 6,0 & 3 & 6,0 & 16 & 32,0 & $<$ \\
\hline Alto & 0 & 0.0 & 21 & 42,0 & 3 & 6,0 & 3 & 6,0 & 27 & 54,0 & 0,05 \\
\hline \multirow[t]{2}{*}{ TOTAL } & 3 & 8.0 & 29 & 58,0 & 10 & 20.0 & 8 & 16,0 & 50 & 100,0 & \\
\hline & \multicolumn{6}{|c|}{ Peso / Edad } & & & & & \\
\hline \multirow[t]{2}{*}{ CONOCIMIENTO } & \multicolumn{2}{|c|}{ Desnutrición } & \multicolumn{2}{|c|}{ Normal } & \multicolumn{2}{|c|}{ Sobrepeso } & \multicolumn{2}{|c|}{ TOTAL } & \multicolumn{2}{|c|}{$x^{2}$} & \\
\hline & $\mathrm{N}^{\circ}$ & $\%$ & $\mathrm{~N}^{0}$ & $\%$ & $\mathrm{~N}^{0}$ & $\%$ & $\mathrm{~N}^{0}$ & $\%$ & & & \\
\hline Baio & 1 & 2,0 & 0 & 0,0 & 6 & 12,0 & 7 & 14,0 & \multirow{4}{*}{\multicolumn{2}{|c|}{$<0,05$}} & \\
\hline Medio & 2 & 4.0 & 11 & 22,0 & 3 & 6,0 & 16 & 32,0 & & & \\
\hline Alto & 0 & 0.0 & 26 & 52,0 & 1 & 2,0 & 27 & 54,0 & & & \\
\hline \multirow[t]{2}{*}{ TOTAL } & 3 & 6.0 & 37 & 74,0 & 10 & 20.0 & 50 & 100,0 & & & \\
\hline & \multicolumn{6}{|c|}{ Talla / Edad } & \multirow{2}{*}{\multicolumn{2}{|c|}{ TOTAL }} & \multirow{2}{*}{\multicolumn{2}{|c|}{$\mathrm{X}^{2}$}} & \\
\hline \multirow[t]{2}{*}{ CONOCIMIENTO } & \multicolumn{2}{|c|}{ Baja } & \multicolumn{2}{|c|}{ Normal } & \multicolumn{2}{|c|}{ Alta } & & & & & \\
\hline & $\mathrm{N}^{\circ}$ & $\%$ & $\mathbf{N}^{\circ}$ & $\%$ & $\mathrm{~N}^{*}$ & $\%$ & $\mathbf{N}^{0}$ & $\%$ & & & \\
\hline Baio & 6 & 12,0 & 0 & 0.0 & 1 & 2,0 & 7 & 14,0 & \multirow{4}{*}{\multicolumn{2}{|c|}{$<0,05$}} & \\
\hline Medio & 7 & 14,0 & 7 & 14,0 & 2 & 4,0 & 16 & 32,0 & & & \\
\hline Allto & 3 & 6.0 & 23 & 46,0 & 1 & 2,0 & 27 & 54,0 & & & \\
\hline TOTAL & 16 & 32,0 & 30 & 60,0 & 4 & 8,0 & 50 & 100,0 & & & \\
\hline
\end{tabular}

Tabla 6. Práctica de madres sobre alimentación y el estado nutricional del Programa Cuna Más de Ciudad Nueva (Tacna - 2019)

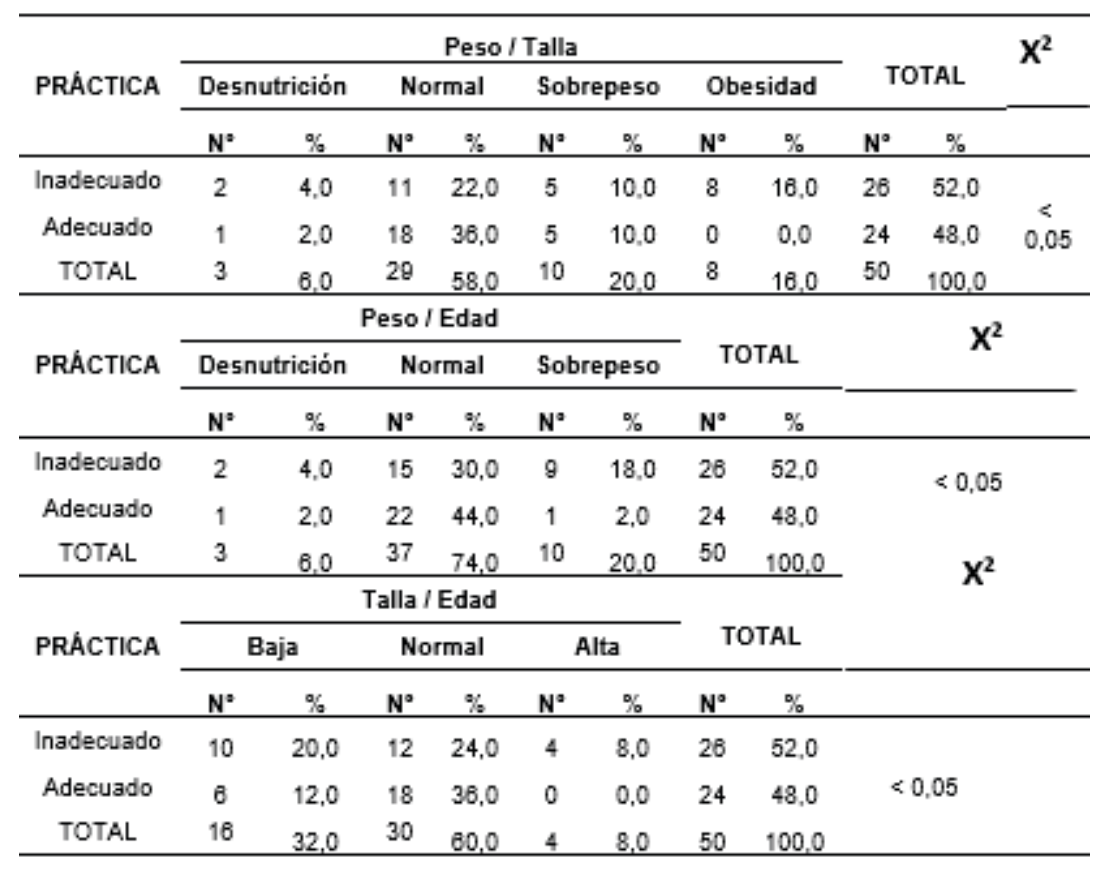

\section{Discusión}

En torno a los seis meses de vida de los niños, los requerimientos de energía y nutrientes de la leche materna no son suficientes, por lo que se hace imprescindible el consumo de alimentos complementarios (4).

Entonces, el infante a partir del año de vida se está desarrollando, y necesita consumir otros alimentos. Si no se ingieren alimentos complementarios en torno de los seis meses, o si son inadecuadamente administrados, el crecimiento del niño puede verse perjudicado.

Los datos sociodemográficos de la madre son algunos factores que podrían determinar el estado nutricional del niño menor de 3 años. La madre cumple un rol fundamental en la alimentación del niño desde su 
nacimiento, y es responsable del cuidado en el crecimiento y desarrollo del niño.

Coincide la presente investigación con el estudio de Zurita M. y Delgado D. (5) "Conocimientos y prácticas de las madres de niños menores de 2 años sobre alimentación complementaria, en un centro de salud, San Miguel 2016 ", donde el $62.5 \%$ de madres señaló tener un nivel de conocimientos alto; $37.5 \%$, un nivel regular, y ninguna señaló tener un nivel bajo.

En cuanto a las prácticas, se diferencia de la presente investigación; el $52.6 \%$ cumplía correctamente con la alimentación de los infantes menores de 2 años; entretanto, el $47.4 \%$ no realizaron las prácticas de alimentación complementaria.

Difiere la presente investigación con la de León R. Francis B. (6), que con el estudio "Conocimiento y prácticas sobre alimentación complementaria en madres con niños de 6 a 24 meses que acuden al Centro de Salud de Revolución, Juliaca - 2015", señala que el $28.6 \%$ de madres tienen un conocimiento bajo; seguido por el $54 \%$, que tienen un conocimiento medio, y, por último, el $17.5 \%$ con un conocimiento alto. Con respecto a la práctica, coincide con la presente investigación; el $74.6 \%$ de las madres presentan prácticas desfavorables, seguido por el $25,4 \%$ que presentan prácticas favorables.

Coincide esta investigación con el trabajo de Vergara T. (7), en el estudio "Nivel de conocimiento de la madre sobre alimentación complementaria y su relación con el estado nutricional del niño de 06 a 12 meses del C.S. Ciudad Nueva, Tacna 2014", donde el mayor número de las madres poseen un nivel medio de conocimientos sobre alimentación complementaria con el $68 \%$.

Difiere la presente investigación con la de Castro K. (8), llamada "Conocimientos sobre alimentación complementaria y su relación con el estado nutricional del niño menor de 2 años. Programa CRED de un C. S. S.J.M 2015 " donde indica que el $28 \%$ de madres poseen un nivel de conocimientos alto; en tanto, el $32 \%$ con nivel de conocimientos medio, y un $40 \%$ con un nivel bajo de conocimiento.

El conocimiento es la suma de hechos y principios que se aprende y retienen a lo largo de la vida como resultado de experiencias, provocando cambios en el proceso del pensamiento, acciones o actividades del individuo. Estos cambios pueden observarse en la conducta y aptitudes del individuo frente a situaciones de la vida diaria; dichas conductas irán cambiando a menudo, conforme aumenta el conocimiento.

Los conocimientos son adquiridos, a través de la experiencia o educación; a nivel teórico o práctico. El entendimiento de un tema se da en un campo particular o general de los hechos; por lo que el conocimiento es, también, la información o conciencia adquirida por la experiencia de un hecho o situación, que ha sucedido dentro de una organización, bien de una forma general o personal (9).

Por lo tanto, el conocimiento de la madre dentro de su rol materno sobre alimentación en el menor de tres años (a través de una correcta orientación e información) mantiene la salud de sus hijos y previene enfermedades.

Las prácticas son las capacidades o experiencias que se obtienen con la realización continua de una actividad. Un conocimiento y práctica adecuada de las madres sobre alimentación en el niño menor de tres años da origen a comportamientos saludables en relación con las costumbres de alimentación, seleccionando alimentos, consistencia y cantidad de las porciones.

Respecto al estado nutricional del niño menor de tres años, coincide la presente investigación con el trabajo de Tasayco A. (10) en el estudio "Nivel de conocimiento sobre alimentación complementaria en madres relacionadas al estado nutricional en infantes menores de 1 año - Centro de Condorillo Alto - Chincha 2016". En este estudio los infantes menores de 1 año presentaron mayormente un estado nutricional normal; según peso/edad alcanzó el 79\%; según talla/edad, el 79\%; y según peso/talla, el $81 \%$; se observa que el estado nutricional de sobrepeso continúa con $18 \%, 14 \%$ y $16 \%$ respectivamente.

Coincide la presente investigación con el trabajo de Ochoa N. (11) en el estudio "Alimentación complementaria y estado nutricional en niños de 6 meses a 2 años atendidos en el Centro de Salud Jorge Chávez, Puerto Maldonado - 2016" al medir el estado de nutrición en los niños; con un $64 \%$ que refleja un estado nutricional normal; seguido de un $24 \%$, desnutrición aguda; el $8 \%$ con desnutrición crónica, y el 4\% con sobrepeso.

Difiere la presente investigación con el trabajo de Castro K. (12) "Conocimientos sobre alimentación complementaria y su relación con el estado nutricional del niño menor de 2 años. Programa CRED de un C.S S.J.M 2015 " sobre el estado nutricional, donde el $46 \%$ presenta desnutrición; el 34\%, un peso normal, y el $20 \%$ presenta sobrepeso.

La nutrición, particularmente en la etapa temprana, constituye uno de los pilares del desarrollo humano y del progreso de las naciones. Tener niños y niñas con excelente estado de nutrición abre, en el presente y en el futuro, una ventana de posibilidades en su capacidad física e intelectual, en la salud, en el desarrollo emocional y social. Por ello es muy importante el poder monitorear el estado nutricional en etapas tempranas de la vida de forma individual para poder medir el progreso del niño, y a nivel nacional para poder medir el avance del país y sus posibilidades (13).

Por lo tanto, el estado nutricional es el equilibrio entre lo que se ingiere y lo que se requiere de nutrientes que 
aportan en la nutrición del infante y, a su vez, restringen su potencial de desarrollo y crecimiento. Al evaluar el estado nutricional se tiene como objetivo verificar el crecimiento y estado de nutrición del niño, y determinar las alteraciones por exceso o defecto; así como distinguir el origen primario o secundario del trastorno nutricional que pueda presentarse.

En este estudio, de acuerdo a la relación peso/ talla se relaciona significativamente $(p=0.011)$, seguido del estado nutricional peso/ edad, se relaciona significativamente $(\mathrm{p}<0.05)$; de igual manera el estado nutricional talla/edad se relaciona significativamente con $(\mathrm{p}=0.003)$.

Difiere con la presente investigación el estudio de Carlo D. (14). En la investigación "Estado nutricional, conocimientos y prácticas alimentarias de niños de 6 a 24 meses de edad que asisten al Centro de Salud Simón Bolívar, Puno 2018". El nivel de conocimiento que tienen las madres con respecto a la alimentación de los niños de 6 a 24 meses de edad, donde el $12.80 \%$ presenta un nivel de conocimiento alto; el $39.40 \%$, un conocimiento medio; y el $47.80 \%$, conocimiento bajo.

Nuestros resultados coinciden con el trabajo de investigación de Vergara T. en el estudio "Nivel de conocimiento de la madre sobre alimentación complementaria y su relación con el estado nutricional del niño de 06 a 12 meses del centro de salud Ciudad Nueva Tacna 2014". Concluyendo que sí existe relación entre el nivel de conocimiento de la madre sobre alimentación complementaria y el estado nutricional del niño; indicadores peso-edad-y peso-talla (15).

En la presente investigación, un alto y medio conocimiento mantiene un estado nutricional normal, un buen conocimiento sobre alimentación, en el menor de tres años, mejora la nutrición dado el rápido crecimiento de los niños, que condiciona elevados requerimientos nutricionales, sumando una capacidad de ingesta limitada en volumen, esta etapa presenta en sí misma una alta vulnerabilidad nutricional como desnutrición, sobrepeso y obesidad; un deficiente conocimiento requiere mayor información para evitar poner en riesgo la nutrición infantil y por ende su estado de salud. Por último, si se considera que el conocimiento de las madres se ve reflejado en el estado nutricional del niño, es imprescindible mejorar el nivel de conocimiento.

Coincide la presente investigación con el trabajo de Mejía T. (16), "Conocimientos maternos sobre alimentación complementaria y su relación con el Estado Nutricional del niño de 6 a 12 meses que asisten al consultorio CRED Centro de Salud Parcona - 2016" concluyendo que sí existe relación entre el nivel de conocimiento de la madre sobre alimentación complementaria y el estado nutricional del niño con indicadores de peso/edad y peso/talla.
La infancia es una etapa de vida idónea para aprender a comer saludablemente. Los hábitos alimentarios adquiridos en este periodo determinan la futura alimentación del niño.

Una adecuada práctica en la alimentación durante los tres primeros años de vida resulta fundamental para el óptimo crecimiento y desarrollo del niño, a su vez las pautas alimentarias aprendidas durante estos años son la base para la constitución de los hábitos alimentarios más tarde en la vida del individuo.

Una práctica adecuada en la alimentación del niño menor de 3 años influye en el estado nutricional, evitando la obesidad, sobrepeso y desnutrición. Una adecuada práctica en la alimentación, durante los dos primeros años de vida, resulta fundamental para el óptimo crecimiento y desarrollo del niño. A su vez, las pautas alimentarias aprendidas durante estos años son más adelante la base para la constitución de los hábitos alimentarios en la vida del individuo.

Se concluye que el mayor porcentaje de madres encuestadas tienen de 21 a 30 años $(56.0 \%)$, seguida de las madres de 31 a 40 años $(26.2 \%)$. Se determinó que el mayor porcentaje de las madres presentaron un alto nivel de conocimiento $(54.0 \%)$, en cuanto a la práctica, la mayoría presentaba prácticas inadecuadas de alimentación en el menor de 3 años $(52 \%)$. Se identificó que la mayoría de los niños presentaba un estado nutricional normal según indicador peso/talla, peso/edad y talla/edad, y se establece la relación significativa entre el conocimiento y práctica, sobre el estado nutricional en niños menores de tres años.

Se recomienda que el personal de enfermería despierte el interés sobre el tema de la alimentación en las madres, informando y demostrando sobre buenos hábitos alimenticios, y la responsabilidad de ser una madre, y las consecuencias irreversibles que produce los malos hábitos alimenticios. El Programa Nacional Cuna Más deberá realizar capacitaciones periódicamente de forma individualizada o grupal a madres, en coordinación con el establecimiento de salud, según el sector al que corresponda, para promover, difundir y fortalecer conocimientos, y buena práctica sobre alimentación en el menor de 3 años; con la finalidad de garantizar una alimentación de calidad, evitando problemas nutricionales en el niño. Realizar trabajos coordinados entre las instituciones a cargo del Ministerio de Salud y las universidades, con el fin de realizar actividades de promoción y prevención para una adecuada alimentación balanceada en el grupo de madres de familias organizadas. El Programa Nacional Cuna Más debería realizar y continuar el seguimiento del estado nutricional en el niño menor de tres años y realizar las referencias respectivas al centro de salud correspondiente. Realizar otras investigaciones con respecto a los factores que modifican el conocimiento, práctica y estado nutricional en la alimentación del niño. 


\section{Referencias bibliográficas}

1. Organización Panamericana de la Salud. Principios de orientación para la alimentación del niño no amamantado entre los 6 y los 24 meses de edad. [Internet]. Washington 2007. [citado en junio del 2019].URL Disponible en https://apps.who.int/iris/bitstream/handle/10665/ 96355/9789275327951_spa.pdf;jsessionid=1 A65 A1ADDD534ED5092E9555CEA7B534?sequen $\mathrm{ce}=1$

2. Organización Mundial de Salud. Alimentación Complementaria. [Internet] 2018. [citado en junio del 2019]. URL Disponible en: https://www.who.int/elena/titles/complementary feeding/es/

3. Dirección regional de salud Tacna. Evaluación anual de la estrategia sanitaria de alimentación y nutrición saludable año 2017. [Internet]. Tacna 2018. [citado en junio del 2019]. URL Disponible en: file://E:/TESIS\%20IRMA\%20AVANCE/Inform e_ES_Alimentacion_y_Nutricion_2017\%20(2).p df

4. Organización mundial de la salud. Salud de la madre, el recién nacido, del niño y del adolescente. Lactancia materna. [Internet]. 2017. [citado en junio del 2019].URL Disponible en Disponible en: https://www.who.int/maternal_child_adolescent/t opics/newborn/nutrition/breastfeeding/es/

5. Zurita M. y Delgado D. Conocimientos y prácticas de las madres de niños menores de 2 años sobre alimentación complementaria, en un centro de salud, San Miguel 2016. [Tesis de grado]. Lima: Universidad Nacional Mayor de San Marcos. 2017. [Internet]. URL Disponible en

http://cybertesis.unmsm.edu.pe/bitstream/handle/ cybertesis/6303/Zurita_em.pdf?sequence $=1$ \&isA llowed $=\mathrm{y}$

6. León r., Francis B. Conocimiento y prácticas sobre alimentación complementaria en madres con niños de 6 a 24 meses que acuden al Centro de Salud de Revolución, Juliaca - 2015. [Tesis de grado]. Puno: Universidad Peruana Unión.2016.[Internet].URL disponible en: https://repositorio.upeu.edu.pe/bitstream/handle/ UPEU/459/Ruth_Tesis_bachiller_2016.pdf?sequ ence $=1 \&$ is Allowed $=y$
7. Vergara T. Nivel de conocimiento de la madre sobre alimentación complementaria y su relación con el estado nutricional del niño de 06 a 12 meses del C.S. ciudad nueva, Tacna 2014. [Tesis de grado]. Tacna: Universidad Nacional Jorge Basadre Grohmann.2014. [Internet] URL Disponible en: http://repositorio.unjbg.edu.pe/bitstream/handle/ UNJBG/391/TG0246.pdf?sequence=1\&isAllowe $\mathrm{d}=\mathrm{y}$

8. Castro K. Conocimientos sobre alimentación complementaria y su relación con el estado nutricional del niño menor de 2 años. Programa CRED de un C.S SJ.M 2015. [Tesis de grado]. Lima: Universidad Nacional Mayor de San Marcos. 2016. [Internet]. URL Disponible en

http://cybertesis.unmsm.edu.pe/bitstream/handle/cyberte sis/4699/Castro_sk.pdf?sequence $=1 \&$ isAllowed= $\mathrm{y}$

9. Vargas D. Definiciones de conocimiento. [Internet]. Método y ciencia. 2015. [citado el 10 de oct 2017]; URL disponible en:https://es.scribd.com/document/266471756/D EFINICIONES-DECONOCIMIENTO-metodo$\mathrm{y}$-ciencia-docx

10. Tasayco A. Nivel de conocimiento sobre alimentación complementaria en madres relacionadas al estado nutricional en niños menores de 1 año centro de Condorillo - Chincha 2016. [tesis de grado]. Lima: Universidad Inca Garcilaso de la Vega. [Internet]. URL Disponible en

http://repositorio.uigv.edu.pe/bitstream/handle/20.500.1 $1818 / 1467 /$ tesis_ana $\% 20$ cecilia $\% 20$ tasayco $\% 20 \mathrm{~h}$ uarote.pdf? sequence $=2 \&$ isallowed $=y$

11. Ochoa N. Chambilla Y. Alimentación complementaria y estado nutricional en niños de 6 meses a 2 años, atendidos en el centro de salud Jorge Chávez, Puerto Maldonado - 2016. Puerto Maldonado. [Tesis de grado]. Puerto Maldonado: Universidad Nacional Amazónica de Madre de Dios.2016. [Internet]. URL. Disponible en: http://repositorio.unamad.edu.pe/bitstream/handl e/UNAMAD/208/004-1-9005.pdf? sequence $=1 \&$ is Allowed $=\mathrm{y}$

12. Castro K. Conocimientos sobre alimentación complementaria y su relación con el estado nutricional del niño menor de 2 años. Programa CRED de un C.S SJ.M 2015. [Tesis de grado]. Lima: Universidad Nacional Mayor de San 
Marcos. 2016. [Internet]. URL Disponible en http:/cybertesis.unmsm.edu.pe/bitstream/handle/cyberte sis/4699/Castro_sk.pdf?sequence=1\&isAllowed=

13. Organización panamericana de la salud. Unidad de nutrición. Principios de orientación para la alimentación complementaria del niño amamantado. [Internet]. Washington DC, 2003. [citado en junio del 2019].URL Disponible en: http://www1.paho.org/hq/dmdocuments/2009/Pri ncipios-orientacion-para-alimentacioncomplementaria-nino-amamantado.pdf

14. Carlo D. Estado Nutricional, conocimientos y prácticas alimentarias de niños de 6 a 24 meses de edad que asisten al Centro de Salud Simón Bolívar, Puno 2018. [tesis de grado]. Puno:

UNIVERSIDAD NACIONAL DEL ALTIPLANO [Internet]. URL Disponible en http://repositorio.unap.edu.pe/bitstream/handle/U NAP/9458/Carlo_Lozada_Dianeth_Soledad.pdf? sequence $=1 \&$ isAllowed $=\mathrm{y}$
15. Vergara T. Nivel de conocimiento de la madre sobre alimentación complementaria y su relación con el estado nutricional del niño de 06 a 12 meses del C.S. ciudad nueva, Tacna 2014. [Tesis de grado]. Tacna: Universidad Nacional Jorge Basadre Grohmann.2014. [Internet] URL Disponible en: http://repositorio.unjbg.edu.pe/bitstream/handle/ UNJBG/391/TG0246.pdf?sequence=1\&isAllowe $\mathrm{d}=\mathrm{y}$

16. Mejía R. Conocimientos Maternos Sobre Alimentación Complementaria y su Relación Con el Estado Nutricional del Niño de 6 a 12 Meses que Asisten al Consultorio Cred Centro Salud Parcona 2016 [Tesis De Grado] Ica: Universidad Nacional San Luis Gonzaga. [Internet]. URL Disponible en http://repositorio.unica.edu.pe/bitstream/handle/ UNICA/2877/44.pdf? sequence $=1$ \&isAllowed $=y$
Correspondencia:

irmarosario15@hotmail.com
Fecha de recepción: 04 de mayo del 2020

Fecha de aceptación: 08 de junio del 2020 